\title{
Association of brain injury and neonatal cytokine response during therapeutic hypothermia in newborns with hypoxic-ischemic encephalopathy
}

\author{
Janet E. Orrock ${ }^{1}$ Karuna Panchapakesan², Gilbert Vezina ${ }^{3,4}$, Taeun Chang ${ }^{4,5}$, Kari Harris ${ }^{5}$, Yunfei Wang ${ }^{4,6}$, \\ Susan Knoblach ${ }^{2,4}$ and An N. Massaro ${ }^{4,7}$
}

\begin{abstract}
BACKGROUND: Cytokines have been proposed as mediators of neonatal brain injury via neuroinflammatory pathways triggered by hypoxia-ischemia. Limited data are available on cytokine profiles in larger cohorts of newborns with hypoxic-ischemic encephalopathy (HIE) undergoing therapeutic hypothermia (TH).
\end{abstract}

METHODS: Serum cytokines interleukin (IL)-1 $\beta,|\mathrm{L}-2,| \mathrm{L}-4$, IL-6, IL-8, IL-10, IL-13, tumor necrosis factor- $\alpha$, and interferon- $\gamma$ were measured in newborns with $\mathrm{HIE}$ at 24 and $72 \mathrm{~h}$ of $\mathrm{TH}$. Differences between infants with favorable (survivors with mild/no magnetic resonance imaging (MRI) injury) vs. adverse outcome (death or moderate/severe MRI injury) were compared using mixed models to adjust for covariates.

RESULTS: Data from 36 term newborns with HIE (favorable outcome: $n=20$, adverse outcome: $n=16$ ) were evaluated. Cytokines IL-1 $\beta, I L-2, I L-6, I L-8, I L-10$, and IL-13 were elevated in the adverse relative to favorable outcome group at $24 \mathrm{~h}$. IL-6 remained significantly elevated in the adverse outcome group at $72 \mathrm{~h}$. IL-6 and IL-10 remained significantly associated with outcome group after controlling for covariates.

CONCLUSION: Inflammatory cytokines are elevated in HIE newborns with brain injury by MRI. In particular, IL-6 and IL-10 were associated with adverse outcomes after controlling for baseline characteristics and severity of presentation. These data suggest that cytokine response may identify infants in need of additional neuroprotective interventions.

$\mathbf{H}$ ypoxic-ischemic encephalopathy (HIE) is a major cause of infant mortality and long-term disability $(1,2)$. Since 2010 , therapeutic hypothermia (TH) has been the standard of care for neonates with moderate-to-severe HIE (3). Despite TH, HIE continues to confer approximately $50 \%$ risk of death or disability (4). Methods to monitor evolution of brain injury at the bedside are needed to identify individuals with inadequate response to $\mathrm{TH}$, signaling the need for potential adjuvant therapies. In particular, brain injury biomarkers that provide insight into pathogenesis can provide specificity to treatment approaches that may further improve outcomes after HIE.

Cytokines and chemokines have been proposed as mediators of neonatal brain injury via neuroinflammatory pathways (5-9). Initially, these pathways were elucidated from animal models of perinatal brain damage in the setting of infection (7). Subsequently, additional triggers of neuroinflammation such as trauma, excitotoxicity, and hypoxia-ischemia have been established (6). Systemic cytokines are often classified as "pro-inflammatory" - such as interleukin (IL)-1 $\beta$, tumor necrosis factor (TNF)- $\alpha$, and interferon (IFN)- $\gamma$-or "anti-inflammatory" - such as IL-4, IL-10, and IL-13. Of note, several cytokines, in particular IL-6, can either propagate or downregulate inflammation, depending on the context (7). Animal $(5,6)$ and human studies $(8,9)$ have also demonstrated specific cytokine trajectories after a hypoxic-ischemic insult. Typically, cytokines peak within $12-24 \mathrm{~h}$ post-insult, but some cytokines have shown a biphasic pattern (8). Given the implicated role of cytokines in the evolution of neonatal brain injury, as well as the dynamic nature of cytokine release after a hypoxic-ischemic insult, investigating serial cytokine levels offers a promising avenue for identifying biomarkers of ongoing brain injury in newborns with HIE.

Limited data are available on cytokine profiles in HIE newborns treated with TH $(8,9)$. This is important since one of the proposed mechanisms of TH includes reduction of inflammation $(10,11)$. Further study is needed to understand the trajectories of inflammatory cytokines in the setting of $\mathrm{TH}$, as well as the relationship between cytokine profiles and brain injury in newborns with HIE.

This study aimed to describe cytokine levels at two key timepoints in the evolution of neonatal HIE: (i) at $24 \mathrm{~h}$ of $\mathrm{TH}$, around the time of secondary energy failure (12) and (ii) at $72 \mathrm{~h}$ of $\mathrm{TH}$, when decisions to initiate rewarming are typically made. We also aimed to evaluate the relationship of neonatal

\footnotetext{
1Pediatric Residency Program, Children's National Health System, Washington, District of Columbia; ${ }^{2}$ Center for Genetic Medicine Research, Children's Research Institute, Washington, District of Columbia; ${ }^{3}$ Department of Diagnostic Imaging and Radiology, Children's National Health System, Washington, District of Columbia; ${ }^{4}$ The George Washington University School of Medicine, Washington, District of Columbia; ${ }^{5}$ Department of Neurology, Children's National Health System, Washington, District of Columbia; ${ }^{6}$ Division of Biostatistics and Study Methodology, Children's National Health System, Washington, District of Columbia; ${ }^{7}$ Division of Neonatology, Children's National Health System, Washington, District of Columbia. Correspondence: An N. Massaro (anguyenm@childrensnational.org) 
cytokine response to magnetic resonance imaging (MRI) evidence of brain injury after HIE. We hypothesized that neonatal cytokine levels would differentiate infants with HIE who died or had severe brain injury from survivors with no-to-mild injury by MRI.

\section{RESULTS}

Of the 93 eligible newborns with HIE admitted to our neonatal intensive care unit (NICU) between September 2010 and March 2014, 82 (88\%) consented to our observational study. A total of 36 newborns with moderate-to-severe HIE with available serum for analysis were included in this study; mean $\pm \mathrm{SD}$ gestational age was $38.8 \pm 1.4 \mathrm{wk}$, mean birth weight was $3.2 \pm 0.7 \mathrm{~kg}$, and $47 \%$ were male. The favorable outcome group consisted of 20 newborns who survived to NICU discharge with minimal-to-no brain injury on MRI. The adverse outcome group was comprised of 16 infants who either died in the neonatal period $(n=7)$ or survived with MRI evidence of moderate-to-severe brain injury $(n=9)$. The adverse outcome group had a lower median 5-min Agpar score (2 vs. 4, $P=0.005)$ and more infants with severe encephalopathy (56 vs $0 \%, P=<0.001)$. No infants had culture-positive sepsis during their NICU stay. Other baseline and clinical characteristics were similar between the groups (Table 1). Serial cytokine levels at the two timepoints of interest ( 24 and $72 \mathrm{~h}$ ) were collected in 26 of 36 patients. The other 10 patients had cytokine determinations at only a single timepoint. At $24 \mathrm{~h}$, there were samples for 32 out of 36 patients (4 patients, including 3 of whom had adverse outcome, had insufficient samples for analysis), while at $72 \mathrm{~h}$, there were samples for 30 out of 36 patients (six patients died between 24 and $72 \mathrm{~h}$ ). Analytic methods to account for missing data (including multiple imputation and last value carry-over approaches) were performed with consistency in analytic results. Analysis results without data interpolation are further presented.

Table 1. Characteristics of the study population

\begin{tabular}{lccc}
\hline & Favorable outcome & Adverse outcome & $P$ value \\
\cline { 2 - 4 } & $(n=20)$ & $(n=16)$ & \\
\hline Birth weight (mean \pm SD kg) & $3.2 \pm 0.9$ & $3.1 \pm 0.5$ & 0.483 \\
$\begin{array}{l}\text { Gestational age } \\
\text { (mean } \pm \text { SD wk) }\end{array}$ & $38.5 \pm 1.5$ & $39.1 \pm 1.2$ & 0.192 \\
Gender ( $n$ \% male) & $11(55)$ & $6(37.5)$ & 0.296 \\
Apgar score & & & \\
$\quad 1$ min & $1(0-4)$ & $1(0-2)^{\mathrm{a}}$ & 0.179 \\
$\quad 5$ min & $4(0-7)$ & $2(0-4)^{\mathrm{a}}$ & $\mathbf{0 . 0 0 5}$ \\
$\begin{array}{l}\text { Presenting pH } \\
\text { Severe encephalopathy } \\
\text { (n,\%) }\end{array}$ & $0.96(6.6-7.42)$ & $6.9(6.59-7.14)$ & 0.2 \\
Chorioamnionitis $(n, \%)$ & $6(38)^{\mathrm{b}}$ & $9(56)$ & $<0.001$ \\
Hour of life at TH initiation & $3: 49(2: 24-5: 52)$ & $4: 16(2: 50-6: 00)$ & 0.124
\end{tabular}

Data are presented as median (range) unless otherwise indicated. Bolded text highlights significant differences between outcome groups.

$\mathrm{TH}$, therapeutic hypothermia.

Data are available for ${ }^{\mathrm{a}} 15$ of $16,{ }^{\mathrm{b}} 16$ of 20 , and ${ }^{\mathrm{c}} 12$ of 16 subjects.
Cytokines IL-1 $\beta$, IL-2, IL-6, IL-8, IL-10, and IL-13 were significantly elevated at $24 \mathrm{~h}$ of $\mathrm{TH}$ in infants with adverse outcomes compared with those with favorable outcomes (Figure 1). At $72 \mathrm{~h}$ of TH, only IL-6 remained significantly elevated in the adverse outcome group. Using a mixed model to adjust for baseline characteristics including gestational age, birth weight, gender, and time of measurement, IL-6 $(P<0.001)$, IL-8 $(P=0.003)$, IL-10 $(P=0.005)$, and IL-13 $(P=0.031)$ remained significantly associated with outcome group. When these models were expanded for clinical characteristics of 5-min Apgar score and presence of severe encephalopathy at presentation, only IL-6 $(P=0.0011)$ and IL-10 $(P=0.0248)$ continued to distinguish outcome groups. Log-IL-6 and log-IL-10 were 1.6 and 2.6 times higher, respectively, in the adverse compared with favorable outcome group after adjusting for clinical covariables. Details of the regression models for IL-6 and IL-10 are presented in Table 2.

Data on placental pathology were available for $28(78 \%)$ of 36 subjects. Chorioamniontis was observed in $9(32 \%)$ of 28 infants. There was no significant difference between the adverse and favorable outcome groups with regards to the frequency of chorioamnionitis ( $25 \mathrm{vs} .38 \%, P=0.483$ ). In the subset of patients with available placental pathology information, IL-6 $(P=0.0017)$ and IL-10 $(P=0.0407)$ remained significantly associated with outcome group after including chorioamnionitis as an additional clinical covariate in the regression models.

\section{DISCUSSION}

In this cohort of 36 newborns, we demonstrate that several inflammatory cytokines hold promise as potential biomarkers of brain injury severity in newborns with HIE treated with TH. In particular, IL-6 and IL-10 consistently distinguished outcome groups across analyses. These differences appear to be the most striking at $24 \mathrm{~h}$ into therapy, potentially offering bedside insight into the therapeutic window by identifying patients with suboptimal response to $\mathrm{TH}$ alone.

While prior studies in animals and humans have demonstrated the association between cytokine profiles with HIE disease severity $(5-9,13-18)$ in the precooling era, our study is the largest to date examining cytokine levels in HIE patients undergoing hypothermia. Two recent studies examined serum cytokine profiles in the setting of hypothermia and have provided important preliminary information on cytokine trajectories and the effects of hypothermia on these trajectories. Chalak et al. (9) evaluated umbilical cord blood and postnatal serial cytokine levels in 20 cooled patients with moderate-to-severe HIE and found that hypothermia and subsequent rewarming had no effect on serial cytokine values. Elevated glial fibrillary acidic protein (GFAP), IL-1, IL-6, IL-8, TNF- $\alpha$, IFN- $\gamma$, and vascular endothelial growth factor at $6-24 \mathrm{~h}$ were associated with abnormal neurological outcomes. In contrast, Jenkins et al. (8) observed a distinct trajectory of certain cytokines (i.e., IL-6, IL-8, and monocyte chemotactic protein-1 (MCP1)), with a biphasic profile observed only in the hypothermia group $(n=20)$. Elevated IL- 6 and MCP-1 levels within $9 \mathrm{~h}$ after birth and low macrophage inflammatory protein 1a (MIP-1a) 


\section{Articles | Orrocket al.}

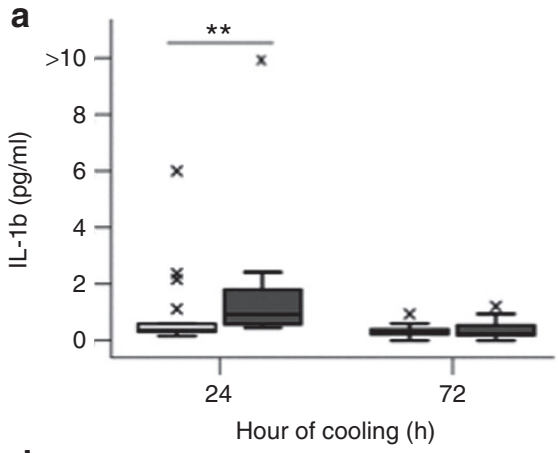

d
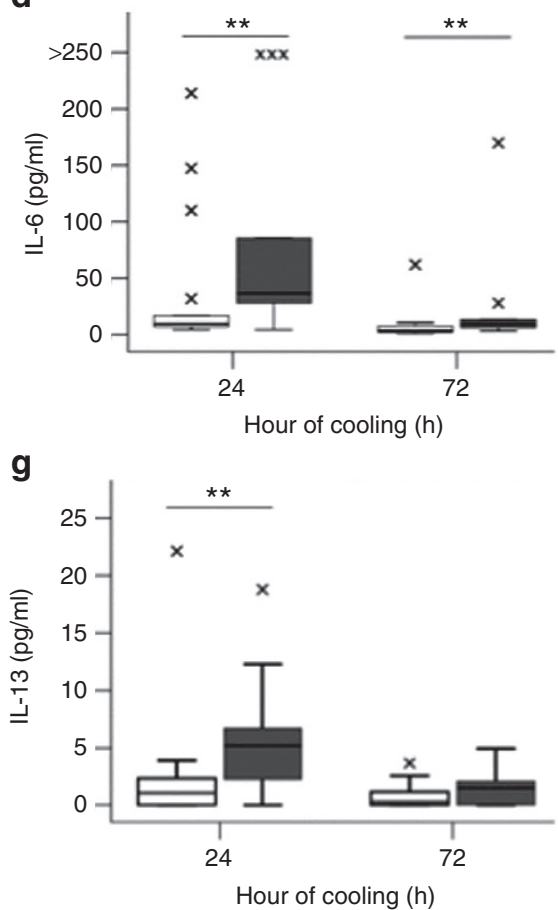

b

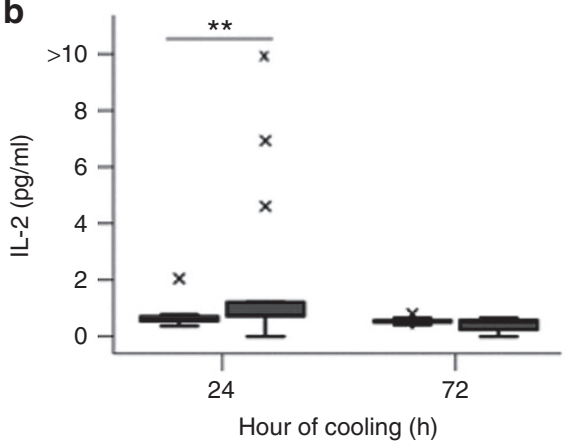

e

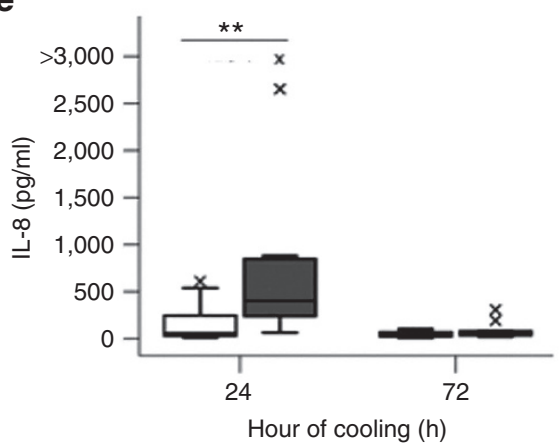

h

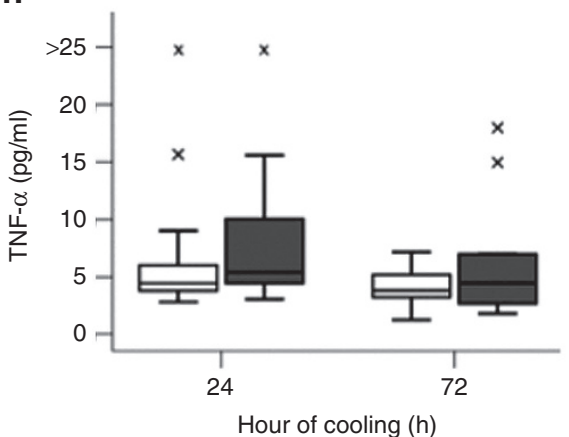

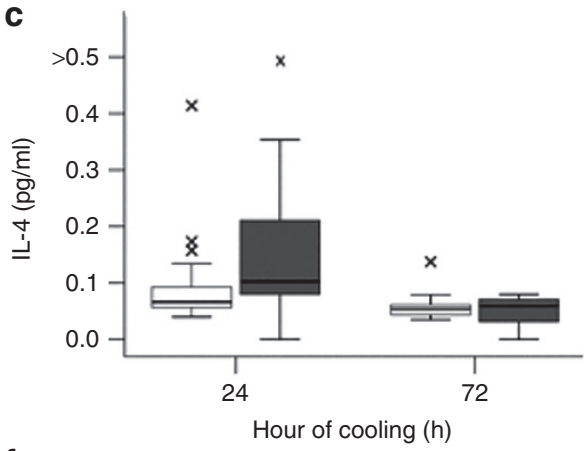

f

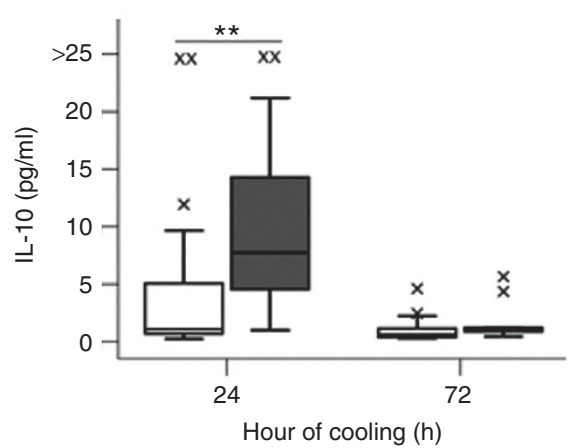

i

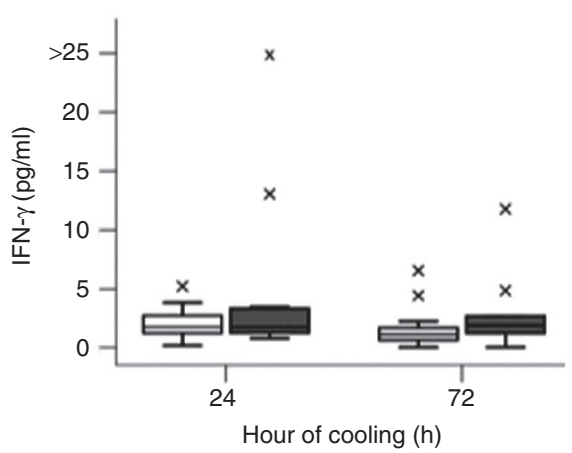

Figure 1. Levels of (a) IL-1b, (b) IL-2, (c) IL-4, (d) IL-6, (e) IL-8, (f) IL-10, (g) IL-13, (h) TNF- $\alpha$, and (i) IFN- $\gamma$ by outcome group. Boxplots represent medians and interquartile ranges. Adverse and favorable outcome groups are represented by shaded and white boxes, respectively. Whiskers represent range with outliers depicted by small asterisks. ${ }^{* *}$ Denotes unadjusted $P<0.05$. IL, interleukin; IFN, interferon; TNF, tumor necrosis factor.

Table 2. Summary of regression models

\begin{tabular}{|c|c|c|c|c|c|c|}
\hline \multirow[b]{2}{*}{ Variable } & \multicolumn{3}{|c|}{ IL-6 } & \multicolumn{3}{|c|}{ IL-10 } \\
\hline & Estimate & SE & $P$ value & Estimate & SE & $P$ value \\
\hline Adverse vs. favorable outcome & 1.6188 & 0.6777 & 0.0248 & 2.5771 & 0.7001 & 0.0011 \\
\hline Age at blood sample & -0.00001 & 0.000063 & 0.8623 & -0.00010 & 0.000065 & 0.1560 \\
\hline Gestational age & -0.3322 & 0.1695 & 0.0612 & -0.1685 & 0.1750 & 0.3450 \\
\hline Birth weight & 0.07808 & 0.3042 & 0.7996 & 0.2860 & 0.3141 & 0.3714 \\
\hline Male gender & 0.1384 & 0.4837 & 0.7771 & 0.7248 & 0.4995 & 0.1592 \\
\hline Apgar at $5 \mathrm{~min}$ & -0.08608 & 0.1129 & 0.4531 & 0.04087 & 0.1166 & 0.7289 \\
\hline Severe encephalopathy & 0.8664 & 0.7336 & 0.2487 & 0.9420 & 0.7583 & 0.2257 \\
\hline
\end{tabular}

Bolded text highlights significant association between outcome group and log-transformed IL-6 and IL-10 levels.

$\mathrm{IL}$, interleukin.

at $60-70 \mathrm{~h}$ of age were associated with death or severely abnormal neurodevelopment at 12 mo of age. Meanwhile, reduction of IL-6, IL-8, and IL-10 from peak levels at $24 \mathrm{~h}$ was observed in infants with better outcomes. These observations highlight the importance of measuring cytokines over time. Equally important is gaining further understanding of the cytokine response in the setting of $\mathrm{TH}$, given this is the current standard of care. While we did not evaluate cytokine trajectories 
in a normothermic group, our study confirms a relationship between inflammatory cytokines and brain injury severity in newborns with HIE undergoing TH.

That IL-6 distinguished outcome groups is not surprising as it has been demonstrated that IL-6 is produced both systemically and in the central nervous system in response to hypoxiaischemia $(5,7)$. Correlation between elevated serum IL- 6 and degree of encephalopathy (19) or impaired neurodevelopmental outcome $(14,15)$ has been observed in studies of both noncooled and cooled infants with HIE. A systematic review of potential brain injury biomarkers identified serum IL- 6 as one of the few independent predictors of adverse outcomes in survivors of HIE (15). That IL-6 is an important biomarker of brain injury after HIE is a consistent finding across ours and prior studies, supporting its role as a putative biomarker that should move forward to more widespread validation. Our study also identified IL-10 as a cytokine that consistently distinguished outcome groups. IL-10 is an anti-inflammatory cytokine, with neuroprotective activity observed in animal studies (7). Although IL-10 has not been as widely investigated as IL- 6 in infants with HIE, Jenkins et al. also reported an association between IL-10 and outcomes after HIE. That other cytokines did not significantly distinguish outcome groups after adjusting for covariates does not eliminate the possibility of their role in the evolution of brain injury after HIE. However, our study highlights these two cytokines as particularly important biomarkers that deserve attention in future studies.

The contribution of inflammation to neonatal brain injury has been well described (7). While one of the proposed mechanisms of hypothermia-mediated neuroprotection has been its anti-inflammatory properties $(10,11)$, it has also been suggested that therapeutic hypothermia may be less effective in newborns with the "double hit" of HIE combined with fetal inflammatory conditions such as chorioamnionitis (20). While we did not observe a difference in rates of reported chorioamnionitis between outcome groups, our study was not aimed or powered to evaluate this relationship. Our ability to elucidate the relationship between chorioamnionitis and neonatal cytokine response was limited by several factors. Placental pathology was not available for all patients. Despite a regional educational initiative, several centers did not retain placental tissue for pathological evaluation in the setting of HIE. While we attempt to retrieve placental tissue for review by our pathologists in all cases of HIE referred to our center, this was not uniformly accomplished. As a result, the determination of chorioamnionitis for the purposes of this study was made by review of maternal clinical records and placental pathology reports from the referral hospital. Thus, we acknowledge the risk for misclassification in this retrospective study. Despite these limitations, we observed that the relationship between IL-6 and IL-10 with brain injury was significant independent of the presence of chorioamnionitis. This suggests that the neonatal cytokine response can be linked to brain injury in the absence of systemic inflammatory conditions, likely reflecting endogenous neuroinflammation leading to secondary injury after hypoxia-ischemia. These data may also support the use of neuroadjuvant therapies that directly target these pathways, such as erythropoietin, which has been shown to decrease the number of IL- $6-$ and TNF- $\alpha$-producing cells and inhibit the synthesis of IL-4, IL-5, and IL-10 (21).

Our study has several limitations. Missing data serve as a potential source of bias, given measurements were not available for both timepoints in all subjects. While we evaluated the effects of this via various statistical methods and found consistent results across analyses, we acknowledge that the effects of missing data remain a concern. It is reassuring that although six patients died before the 72-h blood draw, we were able to include the data of an additional three adverse outcome patients who had data available at $72 \mathrm{~h}$ (even though they did not have 24-h samples) in order to insure that both outcome groups were represented at the two timepoints of interest. Additionally, our relatively small sample size and limited sampling restricted our ability to develop more robust predictive models and to define cytokine trajectories with more temporal granularity. For example, as an outborn level IV NICU, we did not have access to cord blood samples for analysis. However, we analyzed two timepoints that were pragmatically selected based on their clinical and pathophysiological significance. Our early $(24 \mathrm{~h})$ timepoint captures the peak of secondary injury where information on response to treatment may direct the need for adjuvant therapies, whereas the late $(72 \mathrm{~h})$ timepoint coincides with the conventional initiation of rewarming. Evaluating cytokine levels at these key timepoints when clinical decisions are made drove the design of our study where neonatal blood sampling was limited. While cytokines may peak and have variable predictive utility at alternative timepoints, our study aimed to define levels at decision-making timepoints with clinical relevance. Future studies with more frequent collection of samples during cooling and rewarming will be useful to further define the role of these analytes in clinical decision making.

Another limitation of this study is the use of brain injury on neonatal MRI as a surrogate for a long-term outcome measure. Brain MRI performed during the neonatal period is predictive of neurodevelopmental outcome in infants with HIE (22), and its predictive ability is not affected by treatment with hypothermia (23-25). Even still, neurodevelopmental evaluation remains the gold standard for assessing function in childhood survivors of HIE. Follow-up of this cohort is planned, and ongoing and future work will further evaluate the relationship between neonatal cytokine response and long-term neurodevelopmental outcome in babies with HIE.

\section{METHODS}

\section{Study Subjects}

Patients referred to our level IV NICU for therapeutic hypothermia were approached for enrollment in this prospective observational cohort study evaluating blood-based, imaging and physiological biomarkers of brain injury. This report comprises a subset of enrolled newborns with available serum for cytokine analyses. Eligible newborns were treated with whole-body hypothermia according to the Eunice Kennedy Shriver National Institute of Child Health and Human Development (NICHD) Neonatal Research Network protocol, with inclusion criteria according to established NICHD criteria 


\section{Articles $\mid$ Orrocket al.}

(4) (i.e., gestational age greater than $36 \mathrm{wk}$, birth weight greater than $1800 \mathrm{~g}$, metabolic acidosis on cord blood gas and/or depressed Apgar scores, and signs of moderate-to-severe clinical encephalopathy (26)). Whole-body hypothermia was started within $6 \mathrm{~h}$ of life and maintained at $33.5^{\circ} \mathrm{C}$ by esophageal temperature probe for $72 \mathrm{~h}$ prior to rewarming. The study was approved by the Children's National Health System Institutional Review Board, and written informed consent was obtained from the parent of each participant.

\section{Measurement of Cytokines}

One milliliter of blood specimens were collected into silicone-coated microtainers at 24 and $72 \mathrm{~h}$ of cooling from indwelling umbilical arterial or venous lines. Samples were immediately refrigerated, and then serum was isolated within $24 \mathrm{~h}$ (centrifuged at $500 \mathrm{rpm}$ and supernatants stored at $-70^{\circ} \mathrm{C}$ ) to allow for later assay in bulk. We utilized the commercially available V-PLEX Proinflammatory Panel 1 (Meso Scale Discovery, Rockville, MD) multiplex electrochemiluminescence enzyme-linked immunosorbent assay that detects cytokines IL-1 $\beta$, IL-2, IL-4, IL-6, IL-10, IL-13, TNF- $\alpha$, and IFN- $\gamma$ and chemokine IL- 8 from $50 \mu \mathrm{L}$ aliquots run in duplicate and using the QuickPlex SQ120 Plate Reader (Meso Scale Discovery). High, medium, and low range quality control standards provided by the manufacturer were assayed on each plate. The coefficient of variation for inter- and intra-assay variability was $<7 \%$ for all assays. Raw signals generated by the instrument were analyzed using Discovery Workbench 4.0 Software (Meso Scale Discovery). All values were reported in units of $\mathrm{pg} / \mathrm{ml}$. Assays performed in duplicate were averaged for analysis.

\section{Magnetic Resonance Imaging}

Brain MRI was performed according to clinical protocol at 10-12 d of life on a 3 Tesla scanner (Discovery MR750; GE Healthcare, Milwaukee, WI). Standard anatomic sequences included coronal 3D T1-weighted spoiled gradient-recalled, double acquisition axial SE proton density and T2, axial T2 PROPELLER, axial T2*-weighted susceptibility, coronal T1 FLAIR PROPELLER and axial diffusion weighted images. Images were reviewed by an experienced neuroradiologist (G.V.) blinded to clinical data and biomarker levels. Images were scored according to Barkovich with deep nuclear gray injury assigned a basal ganglia (BG) score ranging $0-4$ and cortical/white matter injury assigned a watershed (WS) score ranging $0-5$ (22). The favorable outcome group consisted of survivors with mild $(B G<3$, WS $<4)$ or no $(B G=0, W S=0)$ evidence of brain injury by MRI; the adverse outcome group consisted of patients who died or had a BG score of at least 3 or a WS score of at least 4 .

\section{Data Collection}

Baseline and presenting characteristics were gathered by accessing maternal and infant medical records from the birth hospital. This included review of placental pathology reports from the referral hospital when available, and presence of inflammatory conditions of the placenta including chorioamnionitis, villitis, and funisitis was noted. Pertinent clinical data were also collected from the infants NICU hospitalization including presence of electrographic seizures and any serious bacterial infection including culture-positive sepsis.

\section{Data Analysis}

Descriptive statistics included means \pm SDs or medians (ranges) for parametric and nonparametric variables, respectively, and frequencies for categorical data. Univariate analyses were performed using Wilcoxon Rank Sum Tests to evaluate differences in individual cytokine levels by outcome group. For multivariate analyses, we utilized mixed models to account for repeated measures in subjects. In order to account for outliers in cytokine values, data were logtransformed for normalization. Outcome group, time of measurement, gestational age, birth weight, and gender were included in each model. Expanded models were developed with clinical covariates including presence of severe encephalopathy at presentation, Apgar score at $5 \mathrm{~min}$ of life, and presence of placental inflammation (i.e., chorioamnionitis). Statistical analysis was performed using SAS 9.3 (SAS Institute, Cary, NC).

\section{STATEMENT OF FINANCIAL SUPPORT}

This work was supported by the Clinical and Translational Science Institute at Children's National (UL1TR000075 and 1KL2RR031987-01), the Intellectual and Developmental Disabilities Research Consortium (NIH P3OHD040677), and the NCMRR-DC Molecular and Functional Outcome Measures in Rehabilitation Medicine Core (NICHD/NINDS 5R24HD050846-08).

Disclosure: The authors have no financial relationships relevant to this article to disclose.

\section{REFERENCES}

1. Liu L, Johnson HL, Cousens S, et al.; Child Health Epidemiology Reference Group of WHO and UNICEF. Global, regional, and national causes of child mortality: an updated systematic analysis for 2010 with time trends since 2000. Lancet 2012;379:2151-61.

2. Dilenge ME, Majnemer A, Shevell MI. Long-term developmental outcome of asphyxiated term neonates. J Child Neurol 2001;16:781-92.

3. Perlman JM, Wyllie J, Kattwinkel J, et al.; Neonatal Resuscitation Chapter Collaborators. Neonatal resuscitation: 2010 International Consensus on Cardiopulmonary Resuscitation and Emergency Cardiovascular Care Science with Treatment Recommendations. Pediatrics 2010;126:e1319-44.

4. Shankaran S, Laptook AR, Ehrenkranz RA, et al.; National Institute of Child Health and Human Development Neonatal Research Network. Whole-body hypothermia for neonates with hypoxic-ischemic encephalopathy. N Engl J Med 2005;353:1574-84.

5. Arvin B, Neville LF, Barone FC, Feuerstein GZ. The role of inflammation and cytokines in brain injury. Neurosci Biobehav Rev 1996;20:445-52.

6. Wang X, Feuerstein GZ. Role of immune and inflammatory mediators in CNS injury. Drug News Perspect 2000;13:133-40.

7. Dammann O, O'Shea TM. Cytokines and perinatal brain damage. Clin Perinatol 2008;35:643-63, v.

8. Jenkins DD, Rollins LG, Perkel JK, et al. Serum cytokines in a clinical trial of hypothermia for neonatal hypoxic-ischemic encephalopathy. J Cereb Blood Flow Metab 2012;32:1888-96.

9. Chalak LF, Sánchez PJ, Adams-Huet B, Laptook AR, Heyne RJ, Rosenfeld CR. Biomarkers for severity of neonatal hypoxic-ischemic encephalopathy and outcomes in newborns receiving hypothermia therapy. J Pediatr 2014;164:468-74.e1.

10. Yanagawa Y, Kawakami M, Okada Y. Moderate hypothermia alters interleukin-6 and interleukin-1alpha reactions in ischemic brain in mice. Resuscitation 2002;53:93-9.

11. Diestel A, Roessler J, Berger F, Schmitt KR. Hypothermia downregulates inflammation but enhances IL- 6 secretion by stimulated endothelial cells. Cryobiology 2008;57:216-22.

12. Johnston MV, Fatemi A, Wilson MA, Northington F. Treatment advances in neonatal neuroprotection and neurointensive care. Lancet Neurol 2011;10:372-82.

13. Shalak LF, Laptook AR, Jafri HS, Ramilo O, Perlman JM. Clinical chorioamnionitis, elevated cytokines, and brain injury in term infants. Pediatrics 2002;110:673-80.

14. Bartha AI, Foster-Barber A, Miller SP, et al. Neonatal encephalopathy: association of cytokines with MR spectroscopy and outcome. Pediatr Res 2004;56:960-6.

15. Ramaswamy V, Horton J, Vandermeer B, Buscemi N, Miller S, Yager J. Systematic review of biomarkers of brain injury in term neonatal encephalopathy. Pediatr Neurol 2009;40:215-26.

16. Volpe JJ. Perinatal brain injury: from pathogenesis to neuroprotection. Ment Retard Dev Disabil Res Rev 2001;7:56-64.

17. Silveira RC, Procianoy RS. Interleukin-6 and tumor necrosis factor-alpha levels in plasma and cerebrospinal fluid of term newborn infants with hypoxic-ischemic encephalopathy. J Pediatr 2003;143:625-9.

18. Chiesa C, Pellegrini G, Panero A, et al. Umbilical cord interleukin-6 levels are elevated in term neonates with perinatal asphyxia. Eur J Clin Invest 2003;33:352-8.

19. Tekgul H, Yalaz M, Kutukculer N, et al. Value of biochemical markers for outcome in term infants with asphyxia. Pediatr Neurol 2004;31:326-32.

20. Wintermark P, Boyd T, Gregas MC, Labrecque M, Hansen A. Placental pathology in asphyxiated newborns meeting the criteria for therapeutic hypothermia. Am J Obstet Gynecol 2010;203:579.e1-9.

21. Strunk T, Härtel C, Temming P, Matzke N, Zimmer J, Schultz C. Erythropoietin inhibits cytokine production of neonatal and adult leukocytes. Acta Paediatr 2008;97:16-20. 
22. Barkovich AJ, Hajnal BL, Vigneron D, et al. Prediction of neuromotor outcome in perinatal asphyxia: evaluation of MR scoring systems. AJNR Am J Neuroradiol 1998;19:143-9.

23. Rutherford M, Ramenghi LA, Edwards AD, et al. Assessment of brain tissue injury after moderate hypothermia in neonates with hypoxic-ischaemic encephalopathy: a nested substudy of a randomised controlled trial. Lancet Neurol 2010;9:39-45.

24. Shankaran S, Barnes PD, Hintz SR, et al.; Eunice Kennedy Shriver National Institute of Child Health and Human Development Neonatal
Research Network. Brain injury following trial of hypothermia for neonatal hypoxic-ischaemic encephalopathy. Arch Dis Child Fetal Neonatal Ed 2012;97:F398-404.

25. Cheong JL, Coleman L, Hunt RW, et al.; Infant Cooling Evaluation Collaboration. Prognostic utility of magnetic resonance imaging in neonatal hypoxic-ischemic encephalopathy: substudy of a randomized trial. Arch Pediatr Adolesc Med 2012;166:634-40.

26. Sarnat HB, Sarnat MS. Neonatal encephalopathy following fetal distress. A clinical and electroencephalographic study. Arch Neurol 1976;33:696-705. 